\title{
Approach by Health Professionals to the Side Effects of Antihypertensive Therapy: Strategies for Improvement of Adherence
}

Grazia Maria Guerra ${ }^{1}{ }^{2}{ }^{2}$, Heno Ferreira Lopes ${ }^{1}$, ELizangela Oliveira Freitas ${ }^{2}$, 3 , Fernanda Marciano Consolim-Colombo ${ }^{1}$, Patricia Silva Lessa ${ }^{4}$, Dante Marcelo Artigas Giorgi ${ }^{1}$, Luiz Aparecido Bortolloto ${ }^{1}$ and Eduardo Moacyr Krieger ${ }^{1}$

1. Hypertension Unit, Heart Institute of Hospital Clinical (InCor-HC, FMUSP), School of Medicine, University of São Paulo, São Paulo 05403-000, SP, Brazil

2. São Camilo University Centers, São Paulo 05022-000, SP, Brazil

3. Institute of Cancer of São Paulo State, São Paulo 01246-000, SP, Brazil

4. Department of Electronics and Systems (DES-UFPE), Federal University of Pernambuco, Recife 50670-901, PE, Brazil

\begin{abstract}
The aim was to determine whether complaints about side effects made by stage III hypertensive patients undergoing antihypertensive therapy lead to adequate blood pressure control. Forty-eight patients were monitored by a nurse every 15 days over the course of 180 days. At baseline, both groups presented similar SBP (systolic blood pressure) (GA, 196 (5)) mm Hg and GB, 189 (6) mm $\mathrm{Hg}$ ) and DBP (diastolic blood pressure) (GA, 122 (3) mm Hg and GB, 121 (4) mm Hg). On day 165, after a progressive decline in blood pressure levels, the two groups differed significantly from each other regarding SBP (GA, -16.9 (24) mm Hg and GB, -40.8 (31) mm $\mathrm{Hg}$ ). At the final follow-up, the patients were allocated to two groups: without complaints (GA) and with (GB) complaints about side effects. Complaining about side effects was a decisive factor for immediate nursing intervention and improved control over BP.
\end{abstract}

Key words: Nursing, hypertension, patient adherence, side effect, ambulatory blood pressure monitoring.

\section{Introduction}

Drug side effects play an important role in compliance and treatment since they may negatively impact adherence behaviors and thus jeopardize health outcomes [1]. By the end of one year, up to $50 \%$ of patients undergoing an antihypertensive treatment are noncompliant, and by the end of 5 years, $85 \%$ are noncompliant [2]. Non adherence results from a number of factors, as demonstrated by reports, including the chronicity of the disease, the complexity and cost of the treatment, the side effects of medication, and the quality of the care offered by health professionals [3, 4].

Corresponding author: Grazia Maria Guerra, RN, Ph.D. research fields: adhesion therapy and care with hypertension prevention.
In clinical practice most health professionals are concerned with identifying markers for good blood pressure control in hypertensive patients. Specific methods have been used for this purpose, such as electronic monitoring devices, pill counts, and self-reporting by means of interviews or self-administered questionnaires.

The present study aimed to determine whether stage III hypertensive patients who are undergoing antihypertensive therapy and complain about side effects have more adequate control over their BP (blood pressure) than noncomplainers when patients are monitored at office visits by a nurse every 15 days throughout a 6-month follow-up.

Patients were given medication by a pharmacist on the health care team, who also did the pill count, and 
they underwent a regular medical evaluation every 90 days.

\section{Methods and Materials}

\subsection{Subjects}

The data herein was retrieved from a more comprehensive study conducted by Guerra-Riccio et al. and published in 2004 [5]. The population of the original study consisted of 100 female and male patients who had primary hypertension, defined as SBP (systolic blood pressure) higher than $140 \mathrm{~mm} \mathrm{Hg}$ and DBP (diastolic blood pressure) higher than 90 mm Hg. They received therapy at a hospital for a 6-month period. These patients were randomly assigned to two groups: group A (48 patients), monitored by a nurse every 15 days, and group B (52 patients), monitored by a nurse twice in the course of the treatment, at day 90 and 180. The former group, group A, comprises the population of the present study.

The 48 hypertensive patients were aged 54 ( \pm 10) years on average and were all from the city of São Paulo. They were outpatients at the Hypertension Unit, Heart Institute (InCor), University of São Paulo Medical School, Brazil, where, over the course of 6 months, they were followed and their arterial blood pressure behavior was observed. At the end of the period, on day 180 , the study population was divided into 2 groups based on whether or not the patients complained about any side effects of the antihypertensive treatment: GA made no complaints about side effects, while GB complained about side effects. Group A included 22 patients, 16 females and 6 males; mean age (SD) of 55 (2) years; 5 of them were white and 17, non white; and their mean BMI (body mass index) was 30 (1) kg/m². Group B included 26 patients, 10 females and 16 males; mean age of 53 (1) years; 7 of them were white and 19, nonwhite; and their mean BMI was 31 (1) $\mathrm{kg} / \mathrm{m}^{2}$. Both groups were monitored by a nurse at office visits every 15 days (12 visits) and contacted a pharmacist every 30 days, when antihypertensive drugs were given to them and pills were counted. Also, on day 90, both groups were followed up by a physician who made the necessary adjustments to the medications for each patient; the physician was blinded to group allocation.

The institutional review board of the Heart Institute, University of São Paulo Medical School, approved the study protocol, and a statement of free and informed consent was signed by all participants.

\subsection{Study Protocol}

At baseline (day zero), the patients were interviewed by a nurse who used a questionnaire to collect personal data as well as data on habits, the development of the disease, any potential risk factors for cardiovascular disease, and medicines being taken. The patients also had their weight and height measured. After lying supine for 5 minutes, their blood pressure was measured 3 times consecutively.

Also on day zero, the patients met with a pharmacist who supplied them with the exact number of pills necessary for the next 30 days and instructed them on the proper use of the medications and on potential adverse effects. The encounter with the pharmacist would be repeated every 30 days.

Adherence was assessed through pill counts; for both groups, the average intake was over $80 \%$. The medication given to the patients was free of cost, a very important point, since the high cost of anti-hypertensive medications can impact behavior adherence.

The follow-up visits were monitored by a nurse followed a regular schedule. On each occasion, therapy adherence was reinforced if necessary and new blood pressure measurements were taken.

To investigate the influence of adverse effects on the hypertensive patients' control of their blood pressure, SBP and DBP measurements were taken of both groups at 15-day intervals after baseline and compared with their baseline (day zero) measurements (Fig. 1).

Pressure was gauged using a mercury sphygmomanometer with a proper size cuff (standard and obese size fit). The values yielded at phases I and V 
of the Korotkoff sounds were recorded and rounded to the nearest $2 \mathrm{~mm} \mathrm{Hg}$ following the Eighth Joint National Committee guideline [6]. In total, 3 readings were taken while a patient lay supine, and the results were considered clinical blood pressure measurements. On days 15 and 180, over a period of 24 hours, blood pressure was assessed with a noninvasive device (SpaceLabs 90207; SpaceLabs Inc., Redmond, Washington, USA) including a properly-sized cuff adjusted to the nondominant arm. Blood pressure readings were taken at 10-minute intervals at daytime and at 20-minute intervals at nighttime.

\subsection{Statistical Analysis}

Analysis of the results was exploratory and was carried out with the Stat Soft (Stat Soft, Inc.; USA) statistical analysis software. Sample size was statistically appropriate and remained the same throughout the study. The Student t-test was employed for comparing the means from the two groups. The means of SBP, DBP, and heart rate variables in the 2 groups were analyzed by the repeated-measures ANOVA. Statistical significance was set at $p<0.05$. Results are displayed as means and standard deviations.

\section{Results}

There were no significant differences between GA (without side effects) and GB (with side effects) concerning to age, gender, race, and biochemical characteristics. However, with respect to the echocardiographic data, the two groups differed significantly in terms of the diastolic diameter variable on the first examination, the zero point, (GA, 47.5 (4) $\mathrm{cm}$, and GB, 53.7 (6) cm; $p=0.004$ ), but not on the second examination, conducted 180 days later (GA, 48.6 (7) cm, and GB, 53 (6) cm; $p=0.067$ ). The diastolic diameter did not influence adherence behavior, because of the absence of a significant difference between the groups at the end of the follow-up. The groups also differed significantly with reference to the ejection fraction variable both on the first examination (GA, 0.73 (0.1)\%, and GB, $0.65(0.1) \% ; p=0.028$ ) and on the second examination (GA, $0.72(0.10) \%$, and Group B, $0.65(0.10) \% ; p=0.035)$ (Table 1). As expected, the difference between the groups remained the same throughout the follow-up; therefore, this variable had no impact on adherence behavior either. It should be emphasized at this point that the drug categories were analyzed according to the frequency of occurrence. Dosage compliance was not analyzed, only emphasizing that it treated patients with stage III.

The antihypertensive treatments of GA and GB at baseline were similar, for there were no statistically significant differences between the two groups as follows: diuretics (GA, 45.4\% and GB, 46.1\%), ACEI (angiotensin-converting enzyme inhibitors) (GA, 59\% and GB, 73\%), alpha-adrenergic antagonists (GA, 63.6\% and GB, 57.6\%), and calcium channel antagonists (GA, 68.1\% and GB, 38.4\%). On day 180, the antihypertensive agents were still similar, with no significant differences between GA and GBas follows: diuretics (GA, 50\% and GB, 50\%), ACEI (GA, 50\% and GB, 80.7\%), alpha-adrenergic antagonists (GA, $63.8 \%$ and GB, 57.6\%), and calcium channel antagonists (GA, 68.1\% and GB, 38.4\%). The drug regimen was adjusted by physicians when patients complained. During the protocol, adjustments were made on average 3 (1) times and 6 (1.1) times in GA and GB, respectively. Office visits to the physician were made three times by every patient, at baseline and on days 90 and 180. At such period, the patient's antihypertensive therapy was reexamined, especially if there were any complaints of side effects. At nursing visits, the support of medical staff was requested for reevaluation if necessary.

Twenty-two patients reported no side effects and 26 reported some side effects early in the protocol. The side effects were, specifically, sexual dysfunction, dizziness, and weakness, and they seemed to play a role in behavior adherence (Table 2). Table 2 shows that 26 patients reported side effects from drugs taken in the 
Table 1 Anthropometric data and biochemical and echocardiographic values of group A (without side effects) and group B (with side effects) on the first and second examinations.

\begin{tabular}{|c|c|c|c|}
\hline Variables & Group A & Group B & $p$-value \\
\hline AGE (YEARS) & $55.1(10)$ & $54.3(9)$ & 0.775 \\
\hline WEIGHT (KG) & $75.3(13)$ & $81.6(18)$ & 0.194 \\
\hline WEIGHT 2 (KG) & $74.9(13)$ & $82.3(17)$ & 0.115 \\
\hline HEIGHT(CM) & $1.6(0.1)$ & $1.6(0.1)$ & 0.186 \\
\hline $\mathrm{BMI} \dagger\left(\mathrm{KG} / \mathrm{M}^{2}\right)$ & $30.4(6)$ & $31.1(5)$ & 0.717 \\
\hline GLUCOSE (MG/DL) & $101.7(16)$ & $103.3(15)$ & 0.749 \\
\hline GLUCOSE 2 (MG/DL) & $104.3(18)$ & $103.7(16)$ & 0.906 \\
\hline CREATININE (MG/DL) & $1.2(0.6)$ & $1.4(0.5)$ & 0.359 \\
\hline CREATININE 2 (MG/DL) & $1.2(0.4)$ & $1.3(0.5)$ & 0.578 \\
\hline URIC ACID (MG/DL) & $6.0(1)$ & $7.2(2)$ & 0.064 \\
\hline URIC ACID 2 (MG/DL) & $7.1(3)$ & $7.2(2)$ & 0.888 \\
\hline TOTAL CHOLESTEROL (MG/DL) & $219.3(51)$ & $210.5(45)$ & 0.574 \\
\hline TOTAL CHOLESTEROL 2 (MG/DL) & $213.5(44)$ & $199.4(37)$ & 0.257 \\
\hline TRIGLYCERIDES (MG/DL) & 141.5(104) & $197.0(132)$ & 0.152 \\
\hline TRIGLYCERIDES 2 (MG/DL) & $143.2(67)$ & $159.0(74)$ & 0.465 \\
\hline SODIUM (MEQ) & $140.6(2)$ & $140.3(2)$ & 0.725 \\
\hline SODIUM 2 (MEQ) & $140.3(2)$ & $140.6(1)$ & 0.604 \\
\hline POTASSIUM (MEQ) & $4.2(0.5)$ & $4.1(0.5)$ & 0.781 \\
\hline POTASSIUM 2 (MEQ) & $4.3(0.4)$ & $4.1(0.4)$ & 0.196 \\
\hline SEPT (CM) & $12.07(3)$ & $11.89(2)$ & 0.847 \\
\hline SEPT 2 (CM) & $11.71(1)$ & $12.55(2)$ & 0.234 \\
\hline POSTERIOR WALL (CM) & $11.79(2)$ & $11.88(2)$ & 0.909 \\
\hline POSTERIOR WALL 2 (CM) & $11.50(1)$ & $12.47(2)$ & 0.162 \\
\hline DIASTOLIC DIAMETER (CM) & $47.53(4)$ & $53.71(6)$ & $0.004^{\#}$ \\
\hline DIASTOLIC DIAMETER 2 (CM) & $48.65(7)$ & $53(6)$ & 0.07 \\
\hline EJECTION FRACTION (\%) & $0.73(0.1)$ & $0.65(0.1)$ & $0.028^{\#}$ \\
\hline EJECTION FRACTION 2 (\%) & $0.72(0.1)$ & $0.65(0.1)$ & $0.035^{\#}$ \\
\hline MASS INDEX (G/M2) & $154(70)$ & $170.59(65)$ & 0.495 \\
\hline MASS INDEX 2 (G/M2) & $157.06(66)$ & $169.50(61)$ & 0.559 \\
\hline
\end{tabular}

Table 2 Side effects reported during follow-up by 26 hypertensive patients taking antihypertensive drugs.

\begin{tabular}{lll}
\hline Effects Reported & $\mathrm{N}$ & $\%$ \\
\hline Sexual dysfunction & 6 & 23.07 \\
Headache & 3 & 11.53 \\
Insomnia & 2 & 7.69 \\
Dizziness & 1 & 3.84 \\
Heartburn & 1 & 3.84 \\
Gastralgia & 1 & 3.84 \\
Hypotension & 1 & 3.84 \\
Cramps & 1 & 3.84 \\
Cough & 1 & 3.84 \\
Confusion & 1 & 4.84 \\
Chest pain on movement & 1 & 3.84 \\
Flushing of the face & 1 & 3.84 \\
Edema & 1 & 3.84 \\
Chest pain & 1 & 3.84 \\
Nocturnal cough & 1 & 3.84 \\
Drowsiness & 1 & 3.84 \\
Nosebleed & 1 & 3.84 \\
Constipation & 1 & 3.84 \\
Total & 26 & 100.00 \\
\hline
\end{tabular}


first few weeks of the treatment, while 22 patients reported no side effects. The complaints most frequently mentioned by the patients were sexual dysfunction (23.07\% (6)), headaches (11.53\% (3)), and insomnia (7.69\% (2)). A total of $23.07 \%$ (6) patients complained on the first nursing visit, $27 \%$ (7) on the second, and $19.23 \%$ (5) on the third.

Arterial blood pressure results:

At baseline, both GA and GB had similar SBP levels (GA, 196 (5) mm Hg and GB, 189 (6) mm Hg) and DBP levels (Group A, 122 (3) mm HG and Group B, 121 (4) mm Hg) (Fig. 1).

Fig. 1 shows the systolic and diastolic BP values for GA and GB at point zero and at every 15 days thereafter throughout the study period (180 days). Both GA and GB have similar SBP levels initially. Blood pressure decreases in both groups, but the reduction has no statistical significance.

The heart rate differences between both groups can
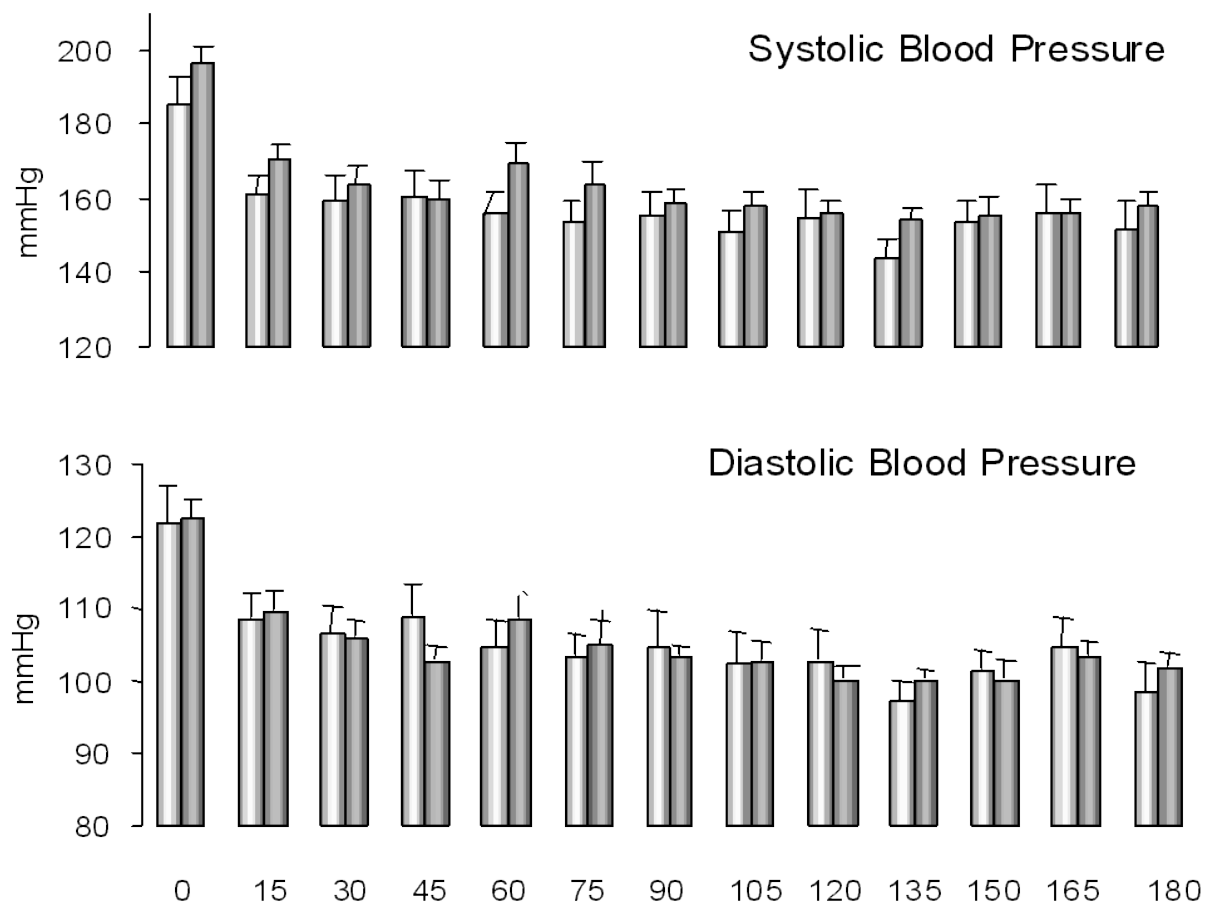

Fig. 1 Mean clinical systolic and diastolic blood pressure levels for group A (white columns, without side effects) and group B (black columns, with side effects) measured at point zero and on subsequent days throughout the study period (total of 180 days).

be seen in Fig. 2. On day 45, the decrease in heart rate in GB is sharper than in GA (-24.4 (23)) bpm and -12.9 (23) bpm; $p=0.008$ ); on day 120 , the difference is even more pronounced (-6.5 (8) bpm and. 0.7 (12) bpm; $p=$ $0.032)$.

Fig. 3 shows the differences between the SBP and DBP levels throughout the study.

There is a significant reduction in SBP and DBP levels starting at baseline in GB (black column). According to sequential analysis, the decline is progressive. The two groups, GA and GB, differ significantly with respect to SBP; the most strong significance seen on day 165 ( $p=0.015)$ (GA, -16.9 (24) $\mathrm{mm} \mathrm{Hg}$ and GB, -40.8 (31) mm Hg).

ABPM (ambulatory blood pressure monitoring):

The results in Table 3 indicate that the mean SBP levels of GA (without side effects) and GB (with side effects), as measured by ABPM, are similar on the first examination during the daytime (GA, 140.7 (21) mm

\section{Systolic Blood Pressure}

Diastolic Blood Pressure 


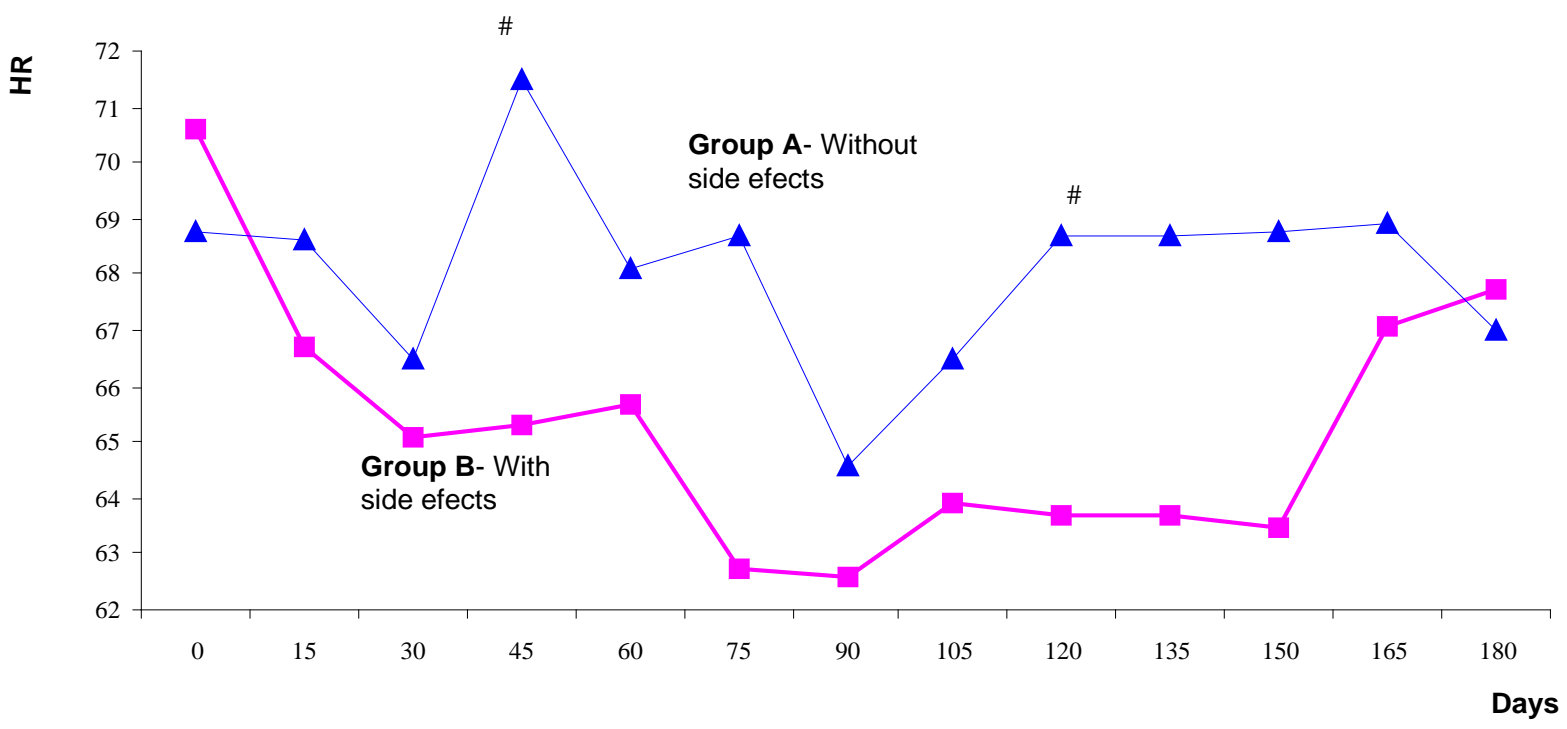

$\# p<0,005$

Fig. 2 Clinical heart rates for group A (blue line, without side effects) and group B (pink line, with side effects) measured at point zero and on subsequent days throughout the study period (total of 180 days).

Systolic Blood Pressure

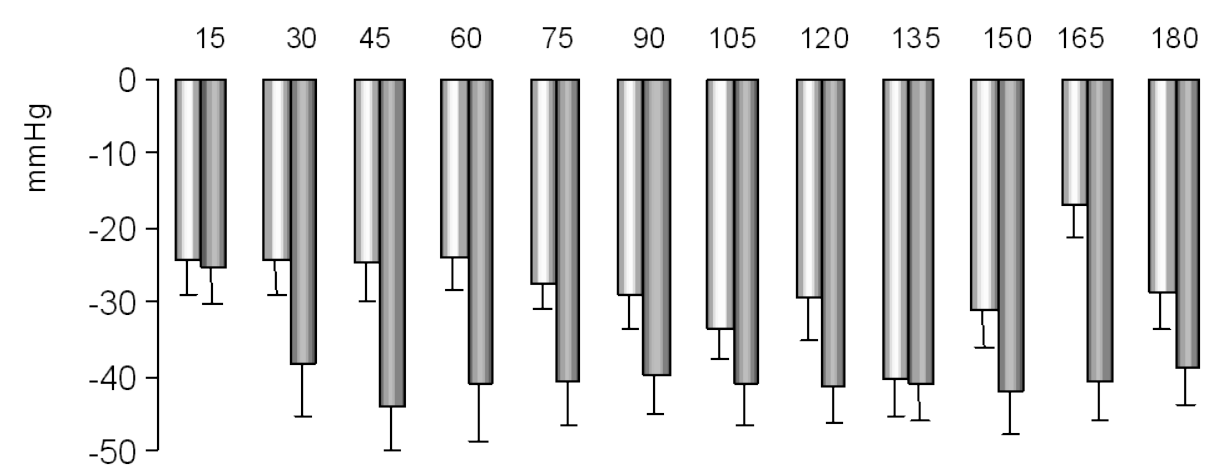

Diastolic Blood Pressure

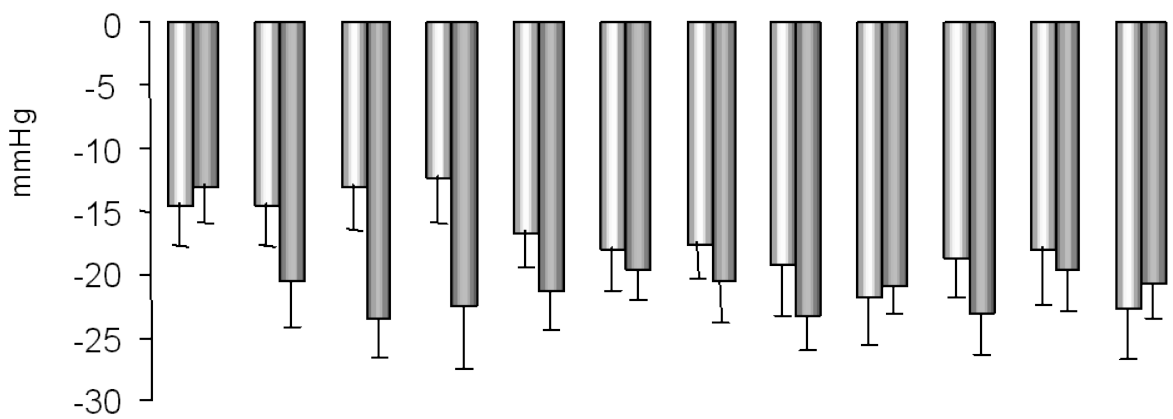

Fig. 3 Differences in SBP and DBP between group A (white columns, without side effects) and group B (black columns, with side effects) over the course of 180 days of treatment. 
Table 3 Ambulatory SBP and DBP measurements taken on day 15 (first exam) and on day 180 (second exam) for both group A (without side effects) and group $B$ (with side effects).

\begin{tabular}{lllll}
\hline & \multicolumn{2}{c}{ Daytime } & \multicolumn{2}{l}{ Nighttime } \\
\cline { 2 - 5 } & $\mathrm{A}$ & $\mathrm{B}$ & $\mathrm{A}$ & $\mathrm{B}$ \\
\cline { 2 - 5 } & SBP/DBP & SBP/DBP & SBP/DBP & SBP/DBP \\
\hline Day 15 (1st exam) & $140.7(21) / 91.2(12)$ & $144.6(18) / 90.6(12)$ & $129.6(21) / 80.5(12)$ & $137.9(29) / 81.7(15)$ \\
Day 180 (2nd exam) & $138.3(23) / 90.0(13)$ & $139.5(20) / 87.2(11)$ & $130.4(33) / 78.3(15)$ & $129.0(30) / 75.9(13)$ \\
\hline
\end{tabular}

SBP = systolic blood pressure; DBP = diastolic blood pressure.

Means (SE) in mmHg.

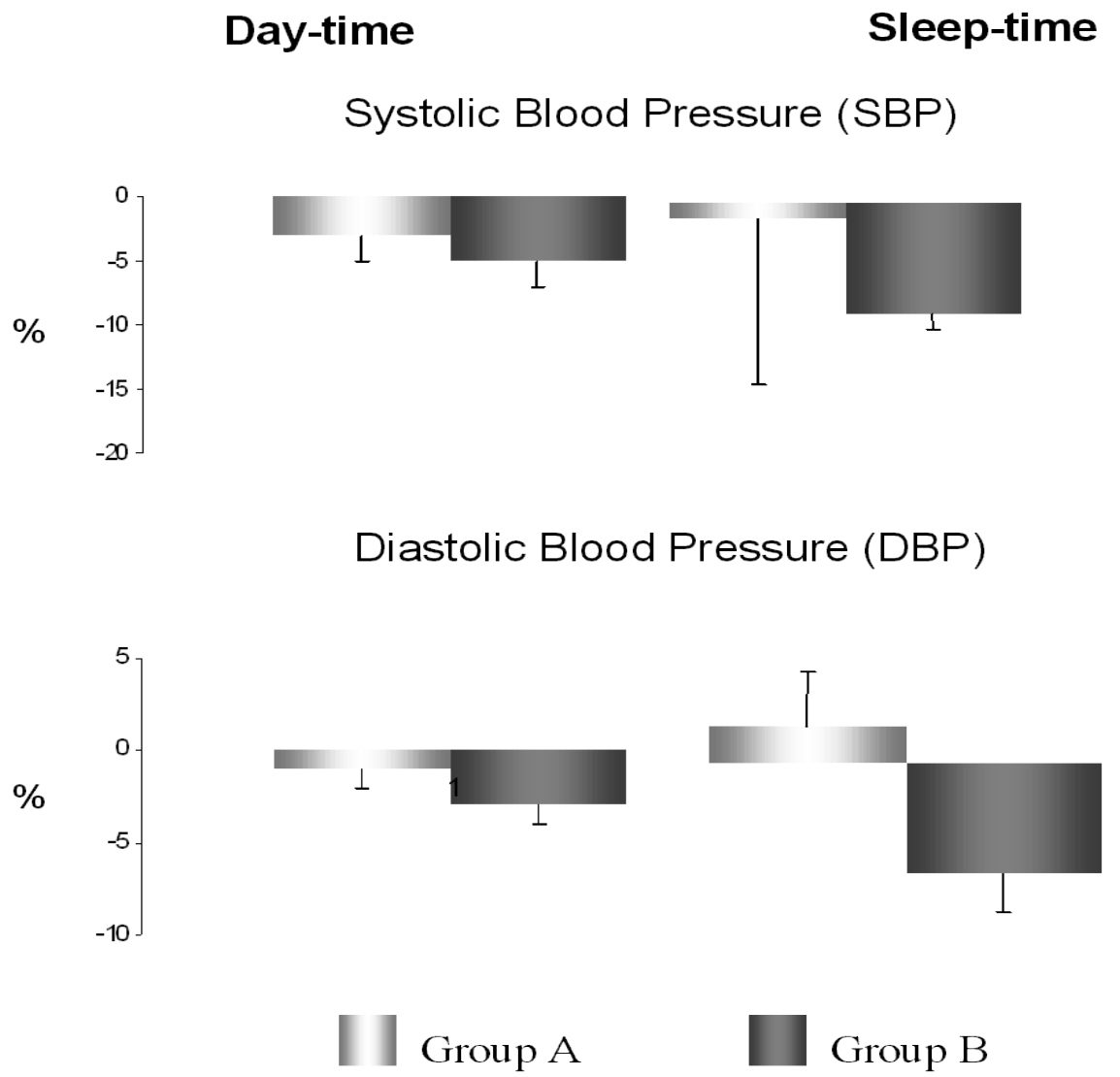

Fig. 4 Comparison of the differences between the 1st and 2nd measurements of systolic blood pressure and diastolic blood pressure found in group A (without side effects) and group B (with side effects) and made during ABPM (ambulatory blood pressure monitoring) at nighttime and daytime.

$\mathrm{Hg}$ and GB, 144.6 (18) $\mathrm{mm} \mathrm{Hg}$ ). The same is true for the DBP levels (GA, 91.2 (12) mm Hg and GB, 90.6 (12) $\mathrm{mm} \mathrm{Hg}$ ). On the first examination at nighttime, the mean SBP levels of both groups, as measured by ABPM, are also similar (GA, 129.6 (21) mm $\mathrm{Hg}$ and GB, 137.9 (29) $\mathrm{mm} \mathrm{Hg}$ ), and so are the mean DBP levels (GA, 80.5 (12) mm Hg and GB, 81.7 (15) mm $\mathrm{Hg}$ ). The standard deviations of the SBP (GA, 3.5 and GB, 11.9; $p=0.012$ ) and DBP (GA, 1.7 and GB, 6.5; $p=0.016$ ) levels in GA differ significantly from those in GB.

Fig. 4 indicates that, on the second examination at daytime, both groups have similar mean SBP levels, as measured by ABPM (GA [without side effects], 138.3 (23) $\mathrm{mm} \mathrm{Hg}$ and GB [with side effects], 139.5 (20) mm $\mathrm{Hg}$ ). The same was observed for the DBP levels (GA, 90.0 (13) mm Hg and GB, 87.2 (11) mm Hg). On the second examination at nighttime, both groups once again have similar mean SBP levels, as measured by ABPM, (GA, 130.4 (33) mm Hg and GB, 129.7 (30) 
mm Hg), and similar DBP levels (GA, 78.3 (15) mm $\mathrm{Hg}$ and GB, 75.9 (13) $\mathrm{mm} \mathrm{Hg}$ ). On the second examination during the daytime, the two groups do not differ significantly from each other as to the SBP levels (GA, 15.5 and GB, 16.4) or the DBP levels (GA, 16.4 and GB, 11.2).

The recorded heart rates on the first examination during the daytime were 70.4 (12) bpm for GA and 66.0 (10) bpm for GB; at nighttime they were 62.3 (7) bpm for GA and 60 (9) bpm for GB. On the second examination at daytime, a significant difference between the two groups was found only for the heart rate (GA, 72.4 (10) bpm and GB, 65.3 (10) bpm; $p=$ 0.046). At nighttime, the differences between the groups had no statistical significance (GA, 6.6 (7.0) bpm and GB, 59.4 (9.7) bpm).

Fig. 4 exhibits the differences in systolic and diastolic blood pressure levels between GA (without side effects) and GB (with side effects) during the daytime and at nighttime and on the 1st and 2nd examinations. No statistically significant differences in SBP levels were found between the groups at daytime (GA, -3 (2) mm Hg and GB, -5 (2) mm Hg) or at nighttime (GA, -1 (12) mm Hg and GB, -8 (1) mm Hg). Also, no differences were found in DBP levels (daytime: GA, -1 (1) mm Hg and GB, -3 (1) mm Hg; nighttime: GA, 2 (3) mm Hg and GB, -6 (1) mm Hg).

\section{Discussion}

In the present study, the presence of side effects was associated with effective adherence to anti-hypertensive treatment through intervention by a nurse. Side effects, specifically sexual dysfunction, dizziness, and weakness, seem to play a role in behavior adherence. All patients, most of them in the Stage III hypertension category [6], were taking antihypertensive medications. Some reports shows that although blood pressure can be brought under control, only half of the patients being medically treated for hypertension are in control of their blood pressure [3, 7-9].

Both study groups in our study had similarly high
BP levels and received similar antihypertensive therapy. In addition, intervention and follow-up were similar. Group B (side effects) had better control of BP as was clearly shown by the follow-up under a nurse's supervision.

There is evidence that the compliance of patients with their anti-hypertensive treatment increases with the presence of nurses on the health care team $[10,11]$. This has been corroborated by the present study's main finding: the hypertensive patients who were frequently monitored by nurses because of the side effects associated with their medical treatment were more effective at managing their blood pressure. Adherence to therapy may be further ensured with improved communication between the patient and the health care provider concerning the risks and benefits of treatment, with customized treatment to better meet patients' needs, and with a careful follow-up. Nurses are often key elements in assessing and managing side effects acquiring importance in the Brazilian basic health units, where they possible can be are major contributors to the patient's ability to control blood pressure and to understand the protocol in Brazil [12].

The time span of eleven weeks was decisive for achieving marked improvement in blood pressure results at follow-up day 165. Although both groups showed decreases in systolic and diastolic BP during follow-up, the SBP levels in GB (with side effects) were significantly lower than those in GA (without side effects) on day 165 . On day 45 , the groups were found to have significantly different heart rates, and on day 120 the difference was even higher. In clinical practice most health professionals are concerned about identifying markers indicative of good blood pressure control in hypertensive patients. Specific methods have been used for this purpose, such as electronic monitoring devices, pill counts, and self-reporting by means of interviews or self-administered questionnaires.

Since BP control was more effective in the group that complained about side effects, we can infer that GB was more susceptible to treatment; however, this 
result is also possibly due to the nurses' effective intervention. This point raises the hypothesis that individuals may have different therapeutic responses, which are provoked by their genetic code and environmental influences.

Frequent nurse monitoring seems to have an additional benefit on the antihypertensive treatment, as was detected differences by clinical BP, although not confirmed by ABPM.

There are many controversies concerning the role of ABPM as an effective method to evaluate adherence behavior [13]. In the present study, clinical BP measurements did not yield the same results as ABPM. In fact, the latter was not sensitive enough to provide accurate measurements for detecting and documenting changes in adherence behavior as already demonstrated in other studies [14]. In our study, differences in SBP and DBP levels were found at daytime and at nighttime between the groups. In GB, the SBP and DBP levels dropped between the first examination and the second one, but the decrease was not significant (Fig. 4). Furthermore, ABPM showed that BP declined less in GA (without side effects) than in GB (with side effects).

Our study has limitations which should be mentioned. First, the sample size was small, possibly limiting our ability to detect significant findings. Second, the white-coat effect was reduced in our study probably due to the fact that office BP was not measured by a physician.

Our data support the hypothesis that the reduction in blood pressure observed in patients with side effects indicate a better therapeutic response. The data suggest that the presence of side effects, when there is a monitoring nurse and immediate intervention, lead to a more effective BP control.

\section{Conclusions}

In conclusion, this study suggests that if the health team does not manage the side effects referred by the patients, they might abandon the treatment, thereby losing effective control of their blood pressure. Further research is needed to confirm this finding and to test other strategies for improved adherence behavior.

\section{What is known about the topic?}

Poor treatment adherence is a major cause of irreversible cardiovascular damage. Adhering to therapy is critical in substantially reducing hypertension-related cardiovascular disease.

\section{What contribution does this study make?}

Many strategies have been tested to improve the hypertensive patient's adherence. However, intervention by a nurse is still considered the cornerstone of the treatment and a main challenge to its effectiveness. This study demonstrates that a patient with side effects can be a marker of compliance.

\section{Acknowledgment}

We thank the Fundação de Amparo a Pesquisa do Estado de São Paulo-FAPESP and the Zerbini Foundation for funding this study.

\section{Disclosure}

The authors declare no conflicts of interest.

\section{References}

[1] Burnier, M. 2006. "Medication Adherence and Persistence as the Cornerstone of Effective Antihypertensive Therapy." American Journal of Hypertension 19: 1190-6.

[2] Andrade, S. E., Gurwitz, J. H., Field, T. S., Kelleher, M., Majumdar, S. R., Reed, G., and Black, R. 2004. "Hypertension Management: The Care Gap between Clinical Guidelines and Clinical Practice.” American Journal of Managed Care 10: 481-6.

[3] Hill, M. N., Bone, L. R., Kim, T. M., Miller, D. J., Dennison, C. R., and Lenine, D. M. 1999. "Barriers to Hypertension Care and Control in Young Urban Black Men.” American Journal of Hypertension 12 (10): 951-8.

[4] Meichenbaum, D., and Turk, D. C. Facilitating Treatment Adherence: The Practitioner's Guide. Plenum Press: New York, 1987.

[5] Guerra-Riccio, G., Giorgi, D., Consolin-Colombo, F., Barreto-Filho, J., Lopes, H., Camargo, A., and Krieger, E. 2004. "Frequent Nurse Visits Decrease White Coat Effect 


\section{Strategies for Improvement of Adherence}

in Stage III Hypertension.” American Journal of Hypertension 17 (6): 523-28.

[6] Chobanian, V., Bakris, G. L., Black, H. R., Cushman, W. C., Green, L. A., Izzo Jr, J. L., et al. 2003. "Seventh report of the Joint National Committee on Prevention, Detection, Evaluation, and Treatment of High Blood Pressure (JNC VII).” Hypertension 42: 1206-52.

[7] Krousel-Wood, M., Hyre, A., Muntner, P., and Morisky, D. 2005. "Methods to Improve Medication Adherence in Patients with Hypertension: Current Status and Future Directions." Current Opinion in Cardiology 20 (4): 296-300.

[8] Samson, R. H. 2004. "Hypertension and the Vascular Patient.” Vascular and Endovascular Surgery 38: 103-19.

[9] Giorgi, D. M. A., and Parati, G. 2007. "Does ABPM Replace Office Blood Pressure Measurement in the Clinical Evaluation of Patients?” Journal of the Brazilian Medical Association 53 (1): 1-12.

[10] Artinian, N. T., Washington, O. G., and Templin, T. N. 2001. "Effects of Home Telemonitoring and Community-Based Monitoring on Blood Pressure Control in Urban African Americans: A Pilot Study.” Heart and
Lung: The Journal of Acute and Critical Care 30 (3): 191-9.

[11] James, P. A., Oparil, S., Carter, B. L., Cushman, W. C., Dennison-Himmelfarb, C., Handler, J., et al. 2014. “2014 Evidence-Based Guideline for the Management of High Blood Pressure in Adults: Report from the Panel Members Appointed to the Eighth Joint National Committee (JNC 8).” JAMA 311 (5): 507-20.

[12] Alexandrer, I. M., and Moore, A. 2007. "Treating Vasomotor Symptoms of Menopause: The Nurse Practitioner's Perspective." Journal of the American Academy of Nurse Practitioners 19: 152-63.

[13] Mancia, G., Facchetti, R., Bombelli, M., Grassi, G., and Sega, R. 2006. "Long-Term Risk of Mortality Associated with Selective and Combined Elevation in Office, Home, and Ambulatory Blood Pressure.” Hypertension 47: 846-53.

[14] Clement, D. L., De Buyzere, M. L., De Bacquer, D. A., de Leeuw, P. W., Duprez, D. A., Fagard, R. H., et al. 2003. "Prognostic Value of Ambulatory Blood Pressure Recordings in Patients with Treated Hypertension.” New England Journal of Medicine 348: 207. 